Polymer Journal, Vol. 9, No. 2, pp 153-159 (1977)

\title{
Anionic Polymerization of tert-Butyl Acrylate
}

\author{
Toshiaki Kitano, Teruo Fujimoto, and Mitsuru Nagasawa \\ Department of Synthetic Chemistry, Nagoya University, \\ Chikusa-ku, Nagoya 464, Japan.
}

(Received November 5, 1976)

\begin{abstract}
The anionic polymerization of tert-butyl acrylate was studied to determine the experimental conditions for preparing poly(acrylic acid) having narrow molecular weight distributions. If the monomer is carefully purified, the monomer can be anionically polymerized with $n$-butyllithium in tetrahydrofuran at $-78^{\circ} \mathrm{C}$. The propagation proceeds by living mechanism, though side reactions seem to occur at the initiation stage. The polymer obtained can be hydrolyzed with hydrobromic acid without degradation.
\end{abstract}

KEY WORDS Anionic Polymerization / Poly(tert-butyl acrylate) / $\alpha, \beta$-Unstaturated Carboxylate / Living Polymerization / Propagation Rate Constant / Hydrolysis of Polyacrylate / Poly(acrylic acid) /

Since the first preparation of polystyrenes with narrow molecular weight distributions by Szwarc, et al., 'with the so-called living polymerization technique, remarkable progress has been achieved in the study of physical properties of linear polymers. In the study of polyelectrolytes, too, it now appears to be essential for the study of their molecular-weight dependent properties to use monodisperse polyelectrolytes. ${ }^{2}$ To change the charge density of polyions, a monodisperse weak polyelectrolyte such as poly(acrylic acid) (PAA) is required. Monodisperse PAA could be prepared by the hydrolysis of monodisperse polyacrylates, if an acrylate monomer can be anionically polymerized by living mechanism.

The purpose of this work is to study the anionic polymerization of tert-butyl acrylate (TBA) in order to determine the experimental conditions for preparing PAA having narrow molecular weight distributions. A method of hydrolysis without chain degradation is also studied.

In the anionic polymerization of $\alpha, \beta$-unsaturated carboxylate monomers such as acrylate, the mechanism of the polymerization is very complicated, due to the possibility of various kinds of side reactions, in comparison with that in the anionic polymerization of hydrocarbon monomers such as styrene. ${ }^{3}$ The problems to be examined include: (1) The carbonyl group may be attacked by the active species, such as initiator and living end. However, the carbonyl group may be protected with a bulky ester group, such as the tert-butyl group. (2) A proton abstraction reaction may occur between the $\alpha$ proton and the active species. In the case of tert-butyl crotonate, it was found that the reaction occurs at the initiation stage but does not occur during the propagation. ${ }^{4,5}$ (3) The purification of acrylate monomer is much more difficult than that of the other $\alpha, \beta$-unsaturated carboxylate monomers or styrenes. (4) The propagation rate in the anionic polymerization of acrylate is extremely fast in comparison with those of methacrylates and crotonates, because of the lack of any electron donating effect of the methyl group and also because of the lack of steric hindrance of the methyl group. A proper polymerization procedure should be found for obtaining the samples which have narrow molecular weight distributions. 


\section{EXPERIMENTAL}

\section{Anionic Polymerization}

TBA monomer, prepared by esterification of acrylic acid with isobutene, was dried with calcium hydride and then distilled in vacuo. TBA was further distilled through a glass tube coated with sodium mirror and finally purified by the same method as used for the purification of tert-butyl crotonate. ${ }^{4}$ That is, a diglyme solution of tritylsodium was dropwise added to the monomer in a dry ice-acetone bath to kill the impurities in the monomer. As soon as the monomer began to polymerize, the monomer was distilled in vacuo using the same glass apparatus as used before ${ }^{4}$ by cooling the reservior in liquid nitrogen. The monomer cannot be warmed owing to its fast polymerization rate. The yield of finally purified monomer is less than 50\%. The final purification was done just before performing the polymerization, since TBA is readily polymerized even in a freezer, if it is so rigorously purified.

The anionic polymerization of TBA was carried out with $n$-butyllithium (n-BuLi) in tetrahydrofuran (THF) at $-78^{\circ} \mathrm{C}$ according to the same procedure as in the anionic polymerization of tert-butyl crotonate. ${ }^{4,5}$ THF solution of the monomer is added to THF solution of $n$-BuLi as instantaneously as possible, so that impurities in the monomer and THF may react with $n$ BuLi at the beginning of the polymerization.

Since the rate of anionic polymerization of TBA is much faster than that of tert-butyl crotonate, a shower nozzle (0.5-mm diameter) and a fritted glass filter, like the ones used in the preparation of monodisperse polystyrene, ${ }^{6}$ were inserted in the same polymerization apparatus as was used for tert-butyl crotonate in order to mix the monomer with the initiator solution homogeneously. Furthermore, to decrease the apparent rate of polymerization, the monomer concentration was lowered below 0.2 $\mathrm{mol} l^{-1}$. If the monomer concentration is higher, a film of polymer is formed on the surface of the polymerizing solution. The other details of the polymerization procedure are the same as reported previously. ${ }^{4}$ Because of the low concentration of monomer in the polymerization mixture, it is essential to purify the solvent and monomer very carefully. After the polymerization, poly(tert-butyl acrylate) (PTBA) was precipitated in a system of methanol and water, and then dried in vacuo. The purification of THF and the preparation of $n$-BuLi were the same as in previous works. ${ }^{4,5}$

The rate of polymerization was measured with a dilatometer to confirm that the polymerization proceeds by living mechanism. The apparatus is the same as used previously. ${ }^{5}$ The diameter of the capillary was about $1.5 \mathrm{~mm}$ and the volume of solution in the dilatometer was $34 \mathrm{~m} l$. The uniformity of the capillary was checked by mercury. An ampule containing the initiator solution was crushed with a magnetic stirrer so that the initiator is mixed with the monomer instantly. By this method, even such a fast polymerization rate could be followed by a dilatometer. If the polymerization rate is of the first order, the following relation must hold over a wide range of conversion: ${ }^{5}$

$$
\log \left(h-h_{1}\right)=-\left(k_{\mathrm{p}} / 2.303\right)[\mathrm{LE}] t+\log \left(h_{0}-h_{1}\right)
$$

where $h, h_{0}$, and $h_{1}$ are the heights of meniscus at time $t$, at zero time, and at the complete conversion, respectively, [LE] is the concentration of living ends, and $k_{\mathrm{p}}$ is the rate constant of propagation. The meniscus of the complete conversion could be determined with a high accuracy. The changes in solution volume at $100-\%$ conversion were about $1-2 \%$ and the changes in height of the capillary were about $15-30 \mathrm{~cm}$ in this dilatometry.

The initiation efficiency $f$, that is, the ratio of the concentration of living ends to that of initiator, was calculated from the initial initiator concentration and the molecular weight of the polymer produced, by the method reported previously. ${ }^{5}$

\section{Hydrolysis of Polyacrylate}

The polyacrylate thus obtained was dissolved in acetone (about $1 \mathrm{~g} / 100 \mathrm{ml}$ ) and warmed up to $60^{\circ} \mathrm{C}$. A small amount of concentrated hydrobromic acid was added to this PTBA solution, but not so much as to precipitate the polymer. After hydrolysis started, a suitable amount of dilute hydrobromic acid solution was occasionally added to the reaction mixture. The hydrolysis reaction was continued homo- 
geneously with stirring under an argon atmosphere. After several hours, the acetone was distilled out and an aqueous solution of sodium hydroxide was added to the remaining polymer solution until the $\mathrm{pH}$ of the solution became greater than 10 . When this viscous solution containing excess $\mathrm{NaOH}$ was poured into methanol, poly(sodium acrylate) was precipitated like a gel. The precipitated poly(sodium acrylate) was purified by using methanol and water, washed with dry methanol, and dried in vacuo. The poly(sodium acrylate) was dissolved again in water and passed through a mixed bed of ion-exchange resins IR-120 and IRA-400 to be converted into PAA.

For characterization, the PAA thus obtained was freeze-dried in vacuo and converted to poly (methyl acrylate) (PMA) by its homogeneous reaction with the methanol complex of boron trifluoride $\mathrm{BF}_{3} \cdot 2 \mathrm{CH}_{3} \mathrm{OH}$ in methanol solution at $65^{\circ} \mathrm{C}$ under an atmosphere of argon. ${ }^{7}$

\section{Characterization of Polymers}

The number-average molecular weights $M_{n}$ of the polymers were determined by osmotic pressure measurements in toluene and in dioxane at $25^{\circ} \mathrm{C}$ with a high speed membrane osmometer type 502 of Hewlett-Packard Co. The weightaverage molecular weights $M_{w}$ were determined by light scattering measurements with a Fica 50 automatic light scattering photometer. The refractive index increments for $546-\mathrm{nm}$ neutral light in dioxane at $25^{\circ} \mathrm{C}$ were determined by a Beckman Spinco Model $\mathrm{H}$ with a cell constructed specially and were found to be $0.0400 \pm 0.0005$ for PTBA and $0.0958 \pm 0.0005$ for PAA. The other values of refractive index increments were available in the literature: $0.0818 \pm 0.0015$ for PTBA in methyl ethyl ketone (MEK) at $25^{\circ} \mathrm{C}$ with $546 \mathrm{~nm}$ light $^{8}$ and 0.097 for PMA in MEK at $30^{\circ} \mathrm{C}$ with $436-\mathrm{nm}$ light. ${ }^{9}$

The sedimentation patterns were obtained in cyclohexane at $35^{\circ} \mathrm{C}$ for PTBA and in acetone at $30^{\circ} \mathrm{C}$ for PMA with a Beckman Spinco Model E. Both are poor solvents.

The degree of hydrolysis was checked by observing $100-\mathrm{MHz}$ NMR spectra of the polymer before and after the hydrolysis, on a JEOL-100 NMR spectrometer. It was also confirmed from the weight and acid concentrations of the sample solution. The acid concentration was determined by titrating the PAA aqueous solution with a standard sodium hydroxide solution on a pH meter of Hitachi-Horiba type H-5; the weight concentration was determined by evaporating the sample solution in a platinum dish.

\section{RESULTS AND DISCUSSION}

First, it is necessary to find an appropriate acrylate monomer and anionic initiator, with which the polymerization would proceed by living mechanism and whose initiation rate would be much faster than the propagation rate. Various preliminary experiments were carried out to find a suitable monomer. The anionic polymerization of methyl acrylate cannot proceed to complete conversion but stops at low conversion. ${ }^{10}$ Acrylates of cyclohexyl, dihydropyranyl, and trimethylsilyl esters were examined as possible monomers. TBA was selected as the best, since we could not find the proper procedures to purify the other acrylates as highly as desired for the present purpose, because of their high radical polymerizability and their unstable properties with regard to temperature and humidity.

It was found that only lithium alkyls give satisfactory results. The other metal alkyls, such as cumylsodium, -potassium, and -cesium, gave polymers with very broad molecular weight distributions. Side reactions, such as carbonyl attack, appear to occur during propagation with these initiators. These results are in agreement with our experience that tert-butyl crotonate cannot be polymerized to high polymers with any other metal alkyls except lithium. ${ }^{4}$

In the anionic polymerization of TBA with $n$-BuLi in THF, it may be assumed that the carbonyl attack would not occur either in the initiation nor in the propagation stage. ${ }^{4,5}$ However, the $\alpha$-proton abstration by $n$-BuLi should occur in the initiation stage. ${ }^{3}$ The initiation efficiency of initiator $f$ was found to be always smaller than unity in this work. For example, in experiment $A$ of Figure 1, about half of the initiators were deactivated $(f=0.5)$. However, it is reasonable to expect that the $\alpha$-proton abstraction would not occur during propagation for the same reasons as discussed in the anionic 
polymerization of tert-butyl crotonate. ${ }^{5}$ That is, the active center during propagation is the enolate anion having a delocalized charge, which is much more inactive than the localized charge of the initiator.

The fact that such side reactions as carbonyl attack and proton abstraction do not occur in the propagation process of TBA can be confirmed by examining the rate of propagation. The results of dilatometry are shown in Figure 1. Good linear relationships between $\log \left(h-h_{1}\right)$ and $t$ were obtained from zero to $90-\%$ conversion. It can be concluded from the figure that the anionic polymerization of TBA proceeds on the first-order rate of polymerization. The apparent rates of propagation $k_{\mathrm{p}}$ estimated from the slopes of the plots were about $(5-7) \times 10^{3}$ $l \mathrm{~mol}^{-1} \mathrm{sec}^{-1}$. However, it is to be noted that the initiation efficiency $f$ was not constant, but

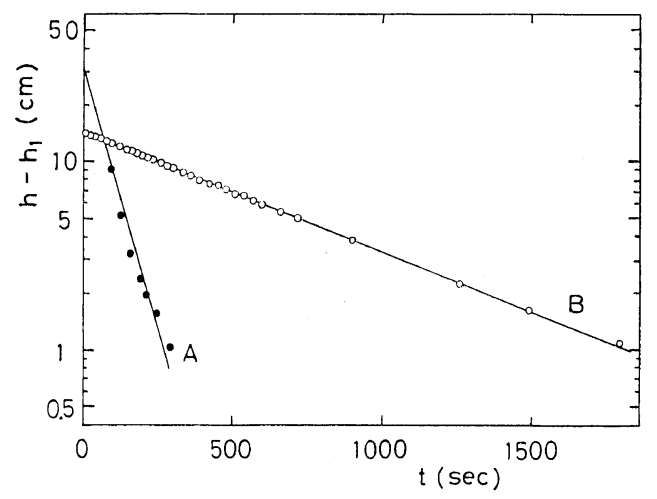

Figure 1. The first-order plots of anionic polymerization of TBA with $n$-BuLi in THF at $-75^{\circ} \mathrm{C}$ : A (filled circles), $[\mathrm{M}]_{0}, 5.4 \times 10^{-1} \mathrm{~mol}^{-1},[\mathrm{I}], 2.3 \times$ $10^{-3} \mathrm{~mol} l^{-1}, \quad[\mathrm{LE}], \quad 1.1 \times 10^{-3} \mathrm{~mol} l^{-1}, \quad k_{\mathrm{p}}, \quad 7 \times 10^{3}$ $l \mathrm{~mol}^{-1} \mathrm{sec}^{-1}, f, 0.50$; $\mathrm{B}$ (open circles), $[\mathrm{M}]_{0}, 2.4 \times$ $10^{-1} \mathrm{~mol} \mathrm{l} l^{-1}$, [I], $6.3 \times 10^{-4} \mathrm{~mol} l^{-1}$, [LE], $1.9 \times 10^{-4}$ $\mathrm{mol} l^{-1}, k_{\mathrm{p}}, 5 \times 10^{3} l \mathrm{~mol}^{-1} \mathrm{sec}^{-1}, f=0.30$.

Table I. Anionic polymerization of TBA with $n$-BuLi in THF at $-78^{\circ} \mathrm{C}$

\begin{tabular}{cccccc}
\hline $\begin{array}{c}\text { Polymeri- } \\
\text { zation }\end{array}$ & $\begin{array}{c}\text { THF } \\
l\end{array}$ & $\begin{array}{c}{[\mathrm{M}]_{0}{ }^{\mathrm{a}}} \\
\mathrm{mol} l^{-1}\end{array}$ & $\begin{array}{c}{[\mathrm{I}]^{\mathrm{b}}} \\
\mathrm{mol}^{-1}\end{array}$ & $\begin{array}{c}{[\mathrm{LE}]^{\mathrm{c}}} \\
\mathrm{mol} l^{-1}\end{array}$ & $f$ \\
\hline I & 1.1 & $1.6 \times 10^{-1}$ & $2.6 \times 10^{-4}$ & $6.8 \times 10^{-5}$ & 0.26 \\
II & 1.6 & $1.2 \times 10^{-1}$ & $2.9 \times 10^{-5}$ & $2.8 \times 10^{-6}$ & 0.10
\end{tabular}

a Initial monomer concentration.

b Initiator concentration.

c Concentration of living ends. decreased with decrease of the initiator concentration. The values of $f$ were $0.50,0.30,0.26$, and 0.10 at $2.2 \times 10^{-3}, 6.3 \times 10^{-4}, 2.6 \times 10^{-4}$, and $2.9 \times 10^{-5} \mathrm{~mol}^{-1}$ of $[\mathrm{I}]$, respectively, as shown in Figure 1 and Table $I$. The monomer concentrations were of the same order in all experiments. Such dependence of $f$ on the initiator concentration $[\mathrm{I}]$ was not observed in the anionic polymerization of tert-butyl crotonate. ${ }^{4}$ This fact may indicate that the monomer TBA

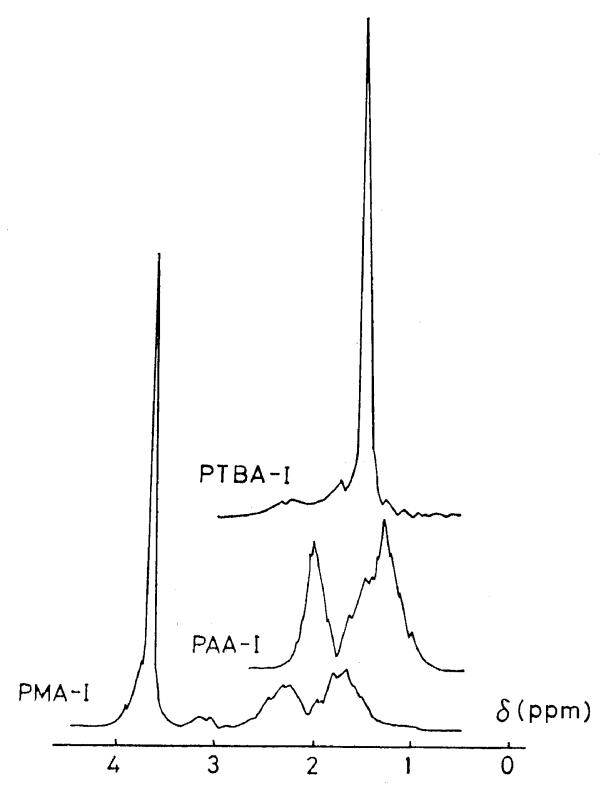

Figure 2. NMR spectra of PTBA-I in $4.7-\mathrm{g} / 100 \mathrm{ml}$ deuterochloroform solution, PAA-I in $4.2-\mathrm{g} / 100 \mathrm{ml}$ deuteromethanol solution, and PMA-I in 4.3-g/100 $\mathrm{m} l$ deuterochloroform solution, at $25^{\circ} \mathrm{C}$ with tetramethylsilane as an internal standard.

Table II. Molecular weights of samples

\begin{tabular}{|c|c|c|c|}
\hline Polymer & $M_{n}^{\mathrm{a}}$ & $M_{w}^{\mathrm{b}}$ & $M_{w} / M_{n}$ \\
\hline TBA-I & $(3.0 \pm 0.2) \times 10^{5}$ & $(4.2 \pm 0.2) \times 10^{5}$ & $1.4 \pm 0$ \\
\hline PAA-I & $\begin{array}{c}(1.6 \pm 0.2) \times 10^{5} \\
{\left[1.7 \times 10^{5}\right]^{\mathrm{c}}}\end{array}$ & $\begin{array}{c}(2.2 \pm 0.2) \times 10^{5} \\
{\left[2.4 \times 10^{5}\right]^{c}}\end{array}$ & \\
\hline TBA-II & - & $(5.4 \pm$ & - \\
\hline PMA-II & - & $\begin{array}{r}(3.3 \pm 0 \\
{[3.6 \times}\end{array}$ & - \\
\hline \multicolumn{4}{|c|}{$\begin{array}{l}\text { a Determined by osmometry. } \\
\text { b Determined by light scattering. } \\
\text { c Molecular weights of PAA calculated from } M \\
\text { and } M_{w} \text { of PTBA, respectively. } \\
\text { d } M_{w} \text { of PMA calculated from } M_{w} \text { of PTBA. }\end{array}$} \\
\hline
\end{tabular}


is not still pure but contains impurities which consume a part of the initiator. Therefore, instantaneous mixing of the monomer with the initiator may be required for the living polymerization of TBA with $n$-BuLi to consume the impurities at the beginning of polymerization.

Two samples of PTBA were eventually pre-

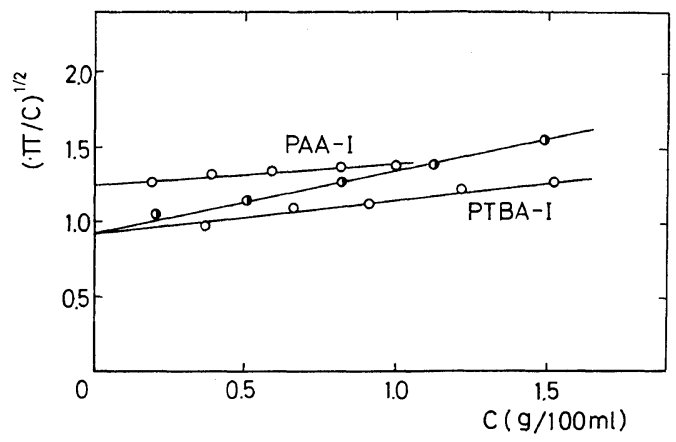

Figure 3. Osmotic pressure measurements of sample I: $\pi$, osmotic pressure $\left(\mathrm{g} / \mathrm{cm}^{2}\right) ; \mathrm{C}$, polymer concentration $(\mathrm{g} / 100 \mathrm{ml})$. Open and half-filled circles denote the data in dioxane and in toluene, respectively, at $25^{\circ} \mathrm{C}$

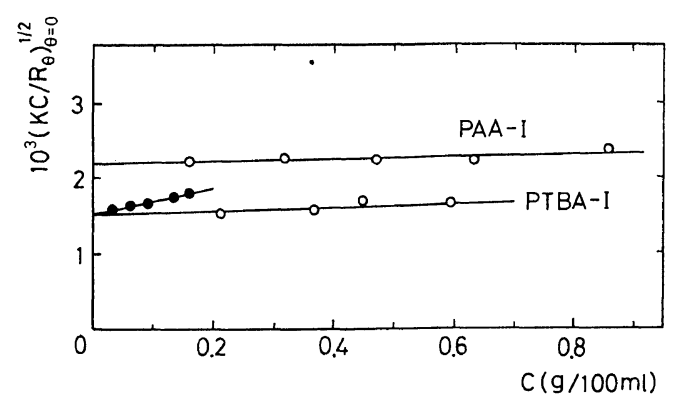

Figure 4. Light-scattering measurements of sample I: $R_{\theta}$, the Rayleigh scattering ratio at angle $\theta ; \mathrm{K}$, the optical constant; $\mathrm{C}$, polymer concentration. Open and filled circles denote the data in dioxane and in MEK, respectively, at $25^{\circ} \mathrm{C}$. pared under the conditions shown in Table $\mathbf{I}$. The conversions were almost $100 \%$ in both cases. PTBA thus obtained was hydrolyzed with $\mathrm{HBr}$. The complete hydrolysis was confirmed by the NMR spectra of PAA, as shown in Figure 2. The ratio of $\alpha$-methine and $\beta$ methylene peaks is 1 to 2 and the peak of tertbutyl ester vanished completely, indicating complete hydrolysis of PTBA. The degree of hydrolysis determined by titration was also $100 \%$ within experimental errors. PAA was methylated and the complete methylation was confirmed by NMR, as shown in Figure 2 .

The molecular weights of the polymers thus obtained are shown in Table II. The results of osmotic pressure and light scattering measurements are shown in Figures 3, 4, and 5, respectively. The number-averaged molecular weight $M_{n}$ of sample II was too high for osmometry. The degrees of polymerization of PTBA, PAA, and also PMA agree within experimental errors. This confirms the lack of chain scission during hydrolysis.

The sedimentation patterns of PAA in dioxane and methanol at $30^{\circ} \mathrm{C}$ were abnormal. The sedimentation patterns of PTBA and PMA are

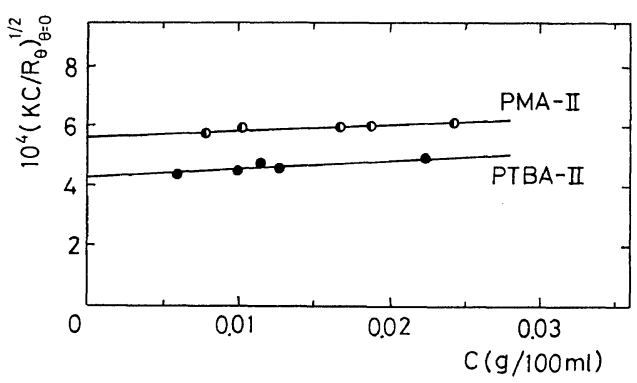

Figure 5. Light-scattering measurements of sample II. Symbols are the same as in Figure 4. Half-filled and filled circles denote the data at $30^{\circ} \mathrm{C}$ and at $25^{\circ} \mathrm{C}$, respectively, in MEK.

Table III. Comparison of propagation rate constants in anionic polymerization of $\alpha, \beta$-unsaturated carboxylates in THF

\begin{tabular}{llccc}
\hline \multicolumn{1}{c}{ Monomer } & \multicolumn{1}{c}{ Initiator } & Temp, ${ }^{\circ} \mathrm{C}$ & $k_{\mathrm{p}}, l \mathrm{~mol}^{-1} \mathrm{sec}^{-1}$ & ref \\
\hline tert -Butyl acrylate & $n$-butyllithium & -75 & $(5-7) \times 10^{3}$ & This work \\
Methyl methacrylate & $\alpha$-Methylstyrylsodium & $-65,-78$ & $1 \times 10^{2}$ & 11 \\
& & -75 & 60 & 12,13 \\
& Cumylcesium & -75 & 80 & 12,13 \\
tert-Butyl crotonate & 2-Methylbutyllithium & -75 & $1 \times 10^{-1}$ & 5 \\
\hline
\end{tabular}


T. Kitano, T. Fujimoto, and M. Nagasawa

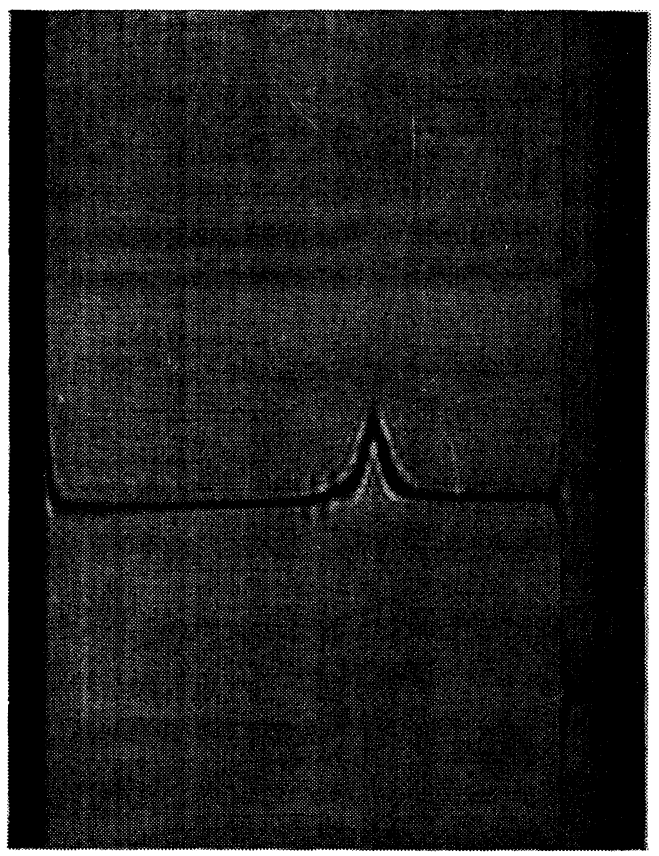

PTBA-I

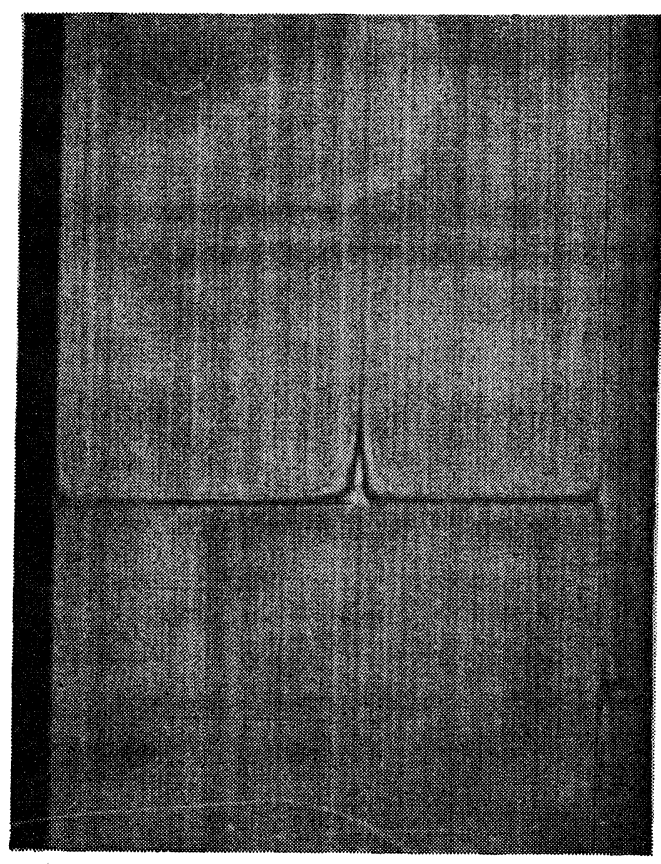

PTBA - I

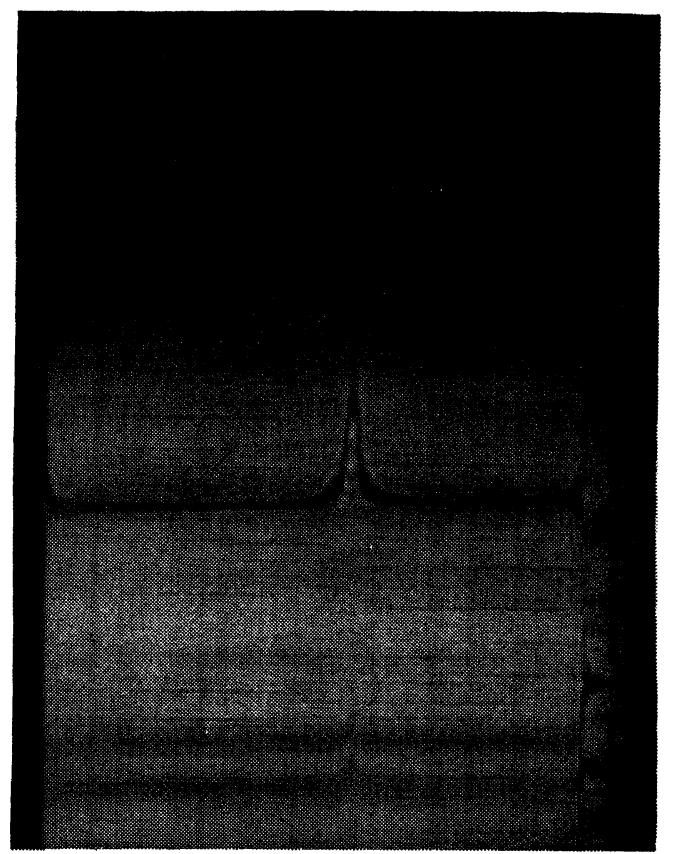

PMA - II

Figure 6. Sedimentation patterns of the samples: speed of rotation, $59780 \mathrm{rpm}$; angle, $70^{\circ}$; PTBA-I in $0.98-\mathrm{g} / 100 \mathrm{ml}$ cyclohexane solution at $35^{\circ} \mathrm{C}$, time, $90 \mathrm{~min}$; PTBA-II in $0.13-\mathrm{g} / 100 \mathrm{ml}$ cyclohexane solution at $35^{\circ} \mathrm{C}$, time, $21 \mathrm{~min}$; PMA-II in 0.21 $\mathrm{g} / 100 \mathrm{ml}$ acetone solution at $30^{\circ} \mathrm{C}$, time, $38 \mathrm{~min}$. 
shown in Figure 6. Unfortunately, it is not possible to determine the molecular weight distributions from these sedimentation patterns since the relationships between sedimentation coefficient and the molecular weight have not yet been determined for PTBA and PMA.

From the data in Table II and the patterns in Figure 6, it is clear that the low molecular weight sample I does not have such a narrow molecular weight distribution as was desired but the high molecular weight sample II has a relatively narrow molecular weight distribution. This result appears to be reasonable, since the time for mixing initiator with monomer is not negligible if the polymerization time is short.

Summarizing the above experimental data, it can be concluded that the anionic polymerization of TBA with $n$-BuLi in THF at $-78^{\circ} \mathrm{C}$ proceeds by living mechanism.

Finally, it is interesting to compare the values of $k_{\mathrm{p}}$ of various $\alpha, \beta$-unsaturated carboxylate monomers in their anionic polymerization. The values of $k_{\mathrm{p}}$ reported so far are listed in Table III. Although the initiators used are not the same in those experiments, the difference in $k_{\mathrm{p}}$ appears to show the effect of the methyl group on the propagation rate, at least qualitatively. That is, the steric hindrance of the methyl group in the $\beta$-position is strong and hence the propagation rate of crotonate is very low. If the methyl group is in the $\alpha$-position, the rate is high due to less steric hindrance. In the ab- sence of the methyl group, the rate is higher.

Acknowledgement. We wish to thank Mr. M. Hirata and Mr. T. Kato for their help in performing the experiments.

\section{REFERENCES}

1. M. Szwarc, M. Levy, and R. Milkovich, $J$. Am. Chem. Soc., 78, 2656 (1956).

2. M. Nagasawa, J. Polym. Sci. Polym. Symp., 49, 1 (1975).

3. T. Tsuruta, “Anionic Polymerization," Kagakudojin, Kyoto, 1973.

4. T. Kitano, T. Fujimoto, and M. Nagasawa, Macromolecule, 7, 719 (1974).

5. T. Kitano, M. Mitsumura, T. Fujimoto, and M. Nagasawa, ibiid., 8, 382 (1975).

6. T. Fujimoto and M. Nagasawa, Polym. J., 7, 397 (1975).

7. L. D. Matcalfe and A. A. Schmitz, Anal. Chem., 33, 363 (1961).

8. R. Jerome and V. Desreux, Eur. Polym. J., 6, 411 (1970).

9. H. Matsuda, K. Yamano, and H. Inagaki, $J$. Polym. Sci. Part A-2, 7, 609 (1969).

10. W. K. Busfield and J. M. Methven, Polymer, 14, 137 (1973).

11. I. Mita, Y. Watanabe, T. Akatsu, and $\mathrm{H}$. Kambe, Polym. J., 4, 271 (1973).

12. G. Löhr and G. V. Schulz, Makromol. Chem., 172, 137 (1973).

13. G. Löhr and G. V. Schulz, Eur. Polym. J., 10, 121 (1974). 